\title{
Extreme values of the Riemann zeta-function on short zero intervals
}

\author{
by
}

\author{
R. R. HALL (York)
}

1. Introduction. We are interested in the distribution of the extreme values taken by the function $|\zeta(1 / 2+i t)|$ between adjacent zeros, conditional on the zero spacing. This study was initiated in [5], and continued by Steuding [7]. Suppose that $\left\{t_{n}: n \in \mathbb{N}\right\}$ denotes the sequence of zeros in $\mathbb{R}^{+}$ counted according to multiplicity and arranged in non-decreasing order, and $N_{0}(T):=\operatorname{card}\left\{n: 0<t_{n} \leq T\right\}$. We write $l_{n}:=t_{n+1}-t_{n}$, and we consider the intervals $\left(t_{n}, t_{n+1}\right)$ satisfying the condition $l_{n} \leq 2 \pi \theta / \log t_{n}$; these are relatively short if $\theta \in \mathbb{R}^{+}$is small, because we expect that $N_{0}(T) \sim(T / 2 \pi) \log T$, so that $l_{n}$ equals approximately $2 \pi / \log t_{n}$ on average, moreover we know unconditionally that $N_{0}(T) \asymp T \log T$. The question arises whether the zetafunction is also relatively small on such intervals, or if it has tall spikes, how often these occur. A complication in this problem is that we do not know the frequency of these short intervals. According to Montgomery's pair correlation conjecture, the number of the intervals specified above with $t_{n} \leq T$ is $\ll \theta^{3} N_{0}(T)$, but actually nothing has been proved in this direction.

Following [5], we define

$$
\begin{aligned}
M_{n} & :=\max \left\{|\zeta(1 / 2+i t)|: t_{n} \leq t \leq t_{n+1}\right\}, \\
M^{(k)}(T, \theta) & :=\sum_{n \leq N}\left\{M_{n}^{2 k}: l_{n} \leq \frac{2 \pi \theta}{\log T}\right\} \quad(k \geq 0) .
\end{aligned}
$$

In the sum (2), $N=N_{0}(T)$. Also $M^{(k)}(T, \infty)$ denotes the sum in which $l_{n}$ is unrestricted. In [5], we showed that

$$
M^{(k)}(T, \theta) \leq H_{k}(\theta)\{1+O(1 / \log T)\} T \log ^{k^{2}+1} T \quad(k=1,2)
$$

where $H_{k}(\theta)$ is an increasing, continuous, bounded function satisfying, in

2000 Mathematics Subject Classification: Primary 11M06. 
the two cases,

$$
\begin{aligned}
& H_{1}(\theta)=\frac{\pi^{3} \theta^{3}}{480} \quad\left(0<\theta \leq \theta_{1}=\frac{5 \sqrt{2}}{\pi \sqrt{3}}\right), \\
& H_{2}(\theta)=\frac{\pi \theta^{3}}{840} \quad\left(0<\theta \leq \theta_{2}=\frac{\sqrt{35}}{\pi \sqrt{3}}\right) .
\end{aligned}
$$

In the range $\theta_{k}<\theta<\infty$, each $H_{k}(\theta)$ is a rather complicated transcendental function, which was evaluated for some typical values and which levels off towards the value obtained in the unrestricted case, respectively:

$$
\begin{aligned}
& H_{1}(\infty)=\frac{5+2 \sqrt{10}}{3 \sqrt{75+60 \sqrt{10}}}=.23200260 \ldots, \\
& H_{2}(\infty)=\frac{28+\sqrt{2086}}{6 \pi^{2} \sqrt{2940+210 \sqrt{2086}}}=.10968770 \ldots / \pi^{2} .
\end{aligned}
$$

The first of the constants in (5) is not far from the best possible since Conrey and Ghosh [2] established that, on the Riemann Hypothesis, actually

$$
M^{(1)}(T, \infty)=\left\{\frac{e^{2}-5}{4 \pi}+o(1)\right\} T \log ^{2} T \quad\left(\frac{e^{2}-5}{4 \pi}=.19011504 \ldots\right)
$$

probably there is a similar formula when $h=2$ with a constant not much smaller than that given in (5). Conrey [1] showed that

$$
\left\{\frac{\sqrt{21}}{90 \pi^{2}}+o(1)\right\} T \log ^{5} T \leq M^{(2)}(T) \leq\left\{\frac{\sqrt{15}}{30 \pi^{2}}+o(1)\right\} T \log ^{5} T,
$$

these constants being $.0509175 \ldots / \pi^{2}$ and $.1290994 \ldots / \pi^{2}$ respectively. The upper bound in (7) is unconditional but the lower bound depends on the hypothesis that Hardy's function $Z(t)$ has only one stationary point in each interval $\left(t_{n}, t_{n+1}\right)$; it is well known that this follows from the Riemann Hypothesis. For small $\theta$ we have $H_{k}(\theta) \ll \theta^{3}$ from (4), and recently Steuding [7] has given a simpler and more transparent proof of this result in the case $k=2$, albeit with a weaker constant $\pi / 140$. He also obtains $H_{1}(\theta) \leq \pi \theta / 6$. I cannot improve my bound for $H_{1}(\theta)$ for any value of $\theta$, but I offer the following result about $\mathrm{H}_{2}(\theta)$.

Theorem 1. The inequality (3) is valid with

$$
H_{2}(\theta)=\frac{\pi^{3} \theta^{5}}{100800} \quad\left(\theta \leq \theta_{2}=\frac{6 \sqrt{14}}{\pi \sqrt{17}}=1.73316908 \ldots\right) .
$$

In the range $\theta_{2} \leq \theta<\infty, H_{2}(\theta)$ increases continuously towards the limit $.1079199 / \pi^{2}$, with some values given in the following table. 
Table 1

\begin{tabular}{ccccc}
\hline$\tau$ & $\psi(\tau)$ & $u$ & $\theta$ & $\pi^{2} H_{2}(\theta)$ \\
\hline 0 & 5 & 0 & $1.7331690 \ldots$ & $.04747811 \ldots$ \\
.1 & $4.88903944 \ldots$ & $.03656627 \ldots$ & $1.7468410 \ldots$ & $.04935892 \ldots$ \\
.2 & $4.58070935 \ldots$ & $.07223527 \ldots$ & $1.7867108 \ldots$ & $.05492413 \ldots$ \\
.3 & $4.12676086 \ldots$ & $.10666825 \ldots$ & $1.8472815 \ldots$ & $.06350647 \ldots$ \\
.4 & $3.57041875 \ldots$ & $.14059931 \ldots$ & $1.9182403 \ldots$ & $.07343739 \ldots$ \\
.5 & $2.93619379 \ldots$ & $.17579172 \ldots$ & $1.9892810 \ldots$ & $.08269191 \ldots$ \\
.6 & $2.24438114 \ldots$ & $.21472579 \ldots$ & $2.0550451 \ldots$ & $.08999126 \ldots$ \\
.7 & $1.52929734 \ldots$ & $.26061358 \ldots$ & $2.1186181 \ldots$ & $.09531191 \ldots$ \\
.8 & $.84806601 \ldots$ & $.31755016 \ldots$ & $2.2024813 \ldots$ & $.09968077 \ldots$ \\
.9 & $.28526769 \ldots$ & $.38748063 \ldots$ & $2.4188151 \ldots$ & $.10440673 \ldots$ \\
1 & 0 & $.44061115 \ldots$ & $\infty$ & $.10791999 \ldots$ \\
\hline
\end{tabular}

The first two columns are explained in the course of the proof below. The improvement obtained over the results in [5] decreases as $\theta$ increases: for example the new bound is better by a factor $7 / 72$ at the old $\theta_{2}=\pi^{-1} \sqrt{35 / 3}$ but at infinity the results are barely distinguishable.

The correct interpretation of this result is not clear. We now have $H_{1}(\theta)$ $\ll \theta^{3}$ and $H_{2}(\theta) \ll \theta^{5}$. If these bounds represented the true orders of magnitude then it would be awkward to match them with the pair correlation conjecture; indeed, $H_{1}(\theta)$ would be essentially the same as the frequency of the short intervals and for this to happen, the behaviour of the $M_{n}$ would have to be more or less independent of $\theta$. An alternative model would be that the $M_{n}$ were usually smaller on these short intervals, with occasional large spikes dominating the sum $M^{(1)}(T, \theta)$. In either scenario $H_{2}(\theta)$ would appear to have to be of the order at least $\theta^{3}$, whereas we know that $H_{2}(\theta) \ll \theta^{5}$.

As in [5] our method involves an inequality relating the maximum modulus of a function on an interval between zeros to certain integral means of the function and some of its derivatives. The new inequality occupies most of the paper. It involves the parameters $\lambda, \mu, \nu$ which ideally would be chosen optimally, however I am not yet able to prove the inequality in the most general case; I choose some parameters that I can cope with, which may not be optimal. This affects the various constants given above and Table 1, but not the exponent 5 of $\theta$.

2. An extremal problem. The results in [5] depended on the following inequality.

Lemma 1. Let $y(x)$ be real-valued on $[a, b], y(a)=y(b)=0$. Suppose that $y$ is twice differentiable, $y^{\prime \prime} \in L^{2}[a, b]$, and that 


$$
\int_{a}^{b} y(x)^{2} d x=A, \quad \int_{a}^{b} y^{\prime}(x)^{2} d x=B, \quad \int_{a}^{b} y^{\prime \prime}(x)^{2} d x=C .
$$

Put $M:=\max \{|y(x)|: a<x<b\}$. Then, for arbitrary $\mu>\lambda>0$, we have

$$
\begin{aligned}
M^{2} \leq & \frac{\lambda^{2} \mu^{2} A+\left(\lambda^{2}+\mu^{2}\right) B+C}{2\left(\mu^{2}-\lambda^{2}\right)} \\
& \times\left\{\frac{1}{\lambda} \tanh \frac{\lambda L}{2}-\frac{1}{\mu} \tanh \frac{\mu L}{2}\right\} \quad(L:=b-a) .
\end{aligned}
$$

There are two useful features here: first that upper bound is linear in $A$, $B$ and $C$, which is essential for the application, and second that the factor involving $L$ on the right is $\ll L^{3}$ for small $L$. The inequality is sharp in the sense that it becomes false in general if any factor $<1$ be introduced on the right-hand side. The question as to whether a sharp bound for $M$ in terms of $A, B, C$ (in the case that (9) is internally consistent) may be derived from (10) by choosing $\lambda$ and $\mu$ in an optimal fashion, is interesting in itself but not relevant to the application to the zeta-function (because we should, in optimizing, lose the linearity in $A, B, C)$.

The idea in [5] was to apply Lemma 1 with $[a, b]=\left[t_{n}, t_{n+1}\right]$ and $y=$ $Z, Z^{2}$ respectively to bound the sums $M^{(1)}(T, \theta)$ and $M^{(2)}(T, \theta)$. I have nothing to add when $k=1$, but observe now that this strategy disregards some information when $k=2$, namely that in this case $y=Z^{2}$ has double zeros at $t_{n}$ and $t_{n+1}$. With this in mind, we look for a version of Lemma 1 containing the extra hypothesis that $y^{\prime}(a)=y^{\prime}(b)=0$, and it emerges that (9) may be usefully supplemented by the equation

$$
\int_{a}^{b} y^{\prime \prime \prime}(x)^{2} d x=D
$$

clearly we need to add the hypothesis that $y^{\prime \prime}$ is differentiable and $y^{\prime \prime \prime} \in$ $L^{2}[a, b]$. Since these moments of $Z(t)$ and its derivatives may all be evaluated, these are acceptable prices. We want an inequality corresponding to Lemma 1 of the following shape, in which the factor $F(\lambda, \mu, \nu ; L)$ on the right is sharp and, for fixed $\lambda, \mu, \nu$, has the property that $F(\lambda, \mu, \nu ; L) \ll L^{5}$ when $L \rightarrow 0$.

CONJECTURAL INEQUALity. Let $y(x)$ be real-valued on $[a, b]$ and $y(a)$ $=y^{\prime}(a)=y(b)=y^{\prime}(b)=0$. Suppose that $y$ is three times differentiable, $y^{\prime \prime \prime} \in L^{2}[a, b]$, and that

$$
\begin{array}{ll}
\int_{a}^{b} y(x)^{2} d x=A, & \int_{a}^{b} y^{\prime \prime}(x)^{2} d x=C, \\
\int_{a}^{b} y^{\prime}(x)^{2} d x=B, & \int_{a}^{b} y^{\prime \prime \prime}(x)^{2} d x=D .
\end{array}
$$


Put $M:=\max \{|y(x)|: a<x<b\}$. Then, for arbitrary $\nu>\mu>\lambda>0$, we have

$$
\begin{aligned}
M^{2} \leq & \left\{\lambda^{2} \mu^{2} \nu^{2} A+\left(\lambda^{2} \mu^{2}+\mu^{2} \nu^{2}+\nu^{2} \lambda^{2}\right) B+\left(\lambda^{2}+\mu^{2}+\nu^{2}\right) C+D\right\} \\
& \times F(\lambda, \mu, \nu ; L),
\end{aligned}
$$

in which $L=b-a$.

In order to define $F$ we introduce the functions

$$
\begin{aligned}
C(\lambda, \mu, \nu ; t):= & \frac{\operatorname{coth}(\lambda t / 2)}{\left(\mu^{2}-\lambda^{2}\right)\left(\nu^{2}-\lambda^{2}\right) \lambda} \\
& +\frac{\operatorname{coth}(\mu t / 2)}{\left(\nu^{2}-\mu^{2}\right)\left(\lambda^{2}-\mu^{2}\right) \mu}+\frac{\operatorname{coth}(\nu t / 2)}{\left(\lambda^{2}-\nu^{2}\right)\left(\mu^{2}-\nu^{2}\right) \nu}, \\
T(\lambda, \mu, \nu ; t):= & \frac{\tanh (\lambda t / 2)}{\left(\mu^{2}-\lambda^{2}\right)\left(\nu^{2}-\lambda^{2}\right) \lambda} \\
& +\frac{\tanh (\mu t / 2)}{\left(\nu^{2}-\mu^{2}\right)\left(\lambda^{2}-\mu^{2}\right) \mu}+\frac{\tanh (\nu t / 2)}{\left(\lambda^{2}-\nu^{2}\right)\left(\mu^{2}-\nu^{2}\right) \nu} .
\end{aligned}
$$

Notice that in each of the sums (14) and (15), there is one negative term, in the middle. As $t \in \mathbb{R}^{+}$increases, $C(\lambda, \mu, \nu ; t)$ decreases from $\infty$, and $T(\lambda, \mu, \nu ; t)$ increases from 0 , toward the common limit

$$
\frac{\lambda+\mu+\nu}{\lambda \mu \nu(\lambda+\mu)(\mu+\nu)(\nu+\lambda)} .
$$

To see the monotonicity property of $T$, observe that the function $\operatorname{sech}^{2} \sqrt{x}$ is a convex function of $x$, which implies that

$$
\operatorname{sech}^{2} \frac{\mu t}{2} \leq \frac{\nu^{2}-\mu^{2}}{\nu^{2}-\lambda^{2}} \operatorname{sech}^{2} \frac{\lambda t}{2}+\frac{\mu^{2}-\lambda^{2}}{\nu^{2}-\lambda^{2}} \operatorname{sech}^{2} \frac{\nu t}{2}
$$

and the result follows on differentiating $T$. A similar argument shows that $C$ is decreasing, actually convex on $\mathbb{R}^{+}$. For this we prove first that the function

$$
y(x):=\sqrt{x} \operatorname{cosech}^{2} \sqrt{x} \operatorname{coth} \sqrt{x}
$$

is a convex function of $x$. We have, noticing that $\sqrt{x} \operatorname{coth} \sqrt{x} \geq 1$ on the second line,

$$
\begin{aligned}
y^{\prime \prime} & (x) \sinh ^{4} \sqrt{x} \\
& =\left\{\frac{1}{2 \sqrt{x}}-\frac{1}{8 x \sqrt{x}}\right\} \sinh 2 \sqrt{x}-\frac{1}{4 x} \cosh 2 \sqrt{x}-\frac{1}{2 x}+\frac{3}{\sqrt{x}} \operatorname{coth} \sqrt{x} \\
& \geq\left\{\frac{1}{2 \sqrt{x}}-\frac{1}{8 x \sqrt{x}}\right\} \sinh 2 \sqrt{x}-\frac{1}{4 x} \cosh 2 \sqrt{x}+\frac{5}{2 x} \\
& =\frac{2}{x}+d_{0}+d_{1} x+d_{2} x^{2}+\cdots \quad \text { (say), }
\end{aligned}
$$


and it emerges that all the coefficients $d_{j}$ are positive. An inequality similar to (17) then establishes that $C^{\prime \prime}(\lambda, \mu, \nu ; t)>0$.

Since $T(\lambda, \mu, \nu ; t)$ is increasing and bounded it cannot be convex: it is intuitive, but we shall not prove, that $T^{\prime \prime}$ has one sign change from positive to negative. We have the expansions

$$
\begin{aligned}
& C(\lambda, \mu, \nu ; t)=\frac{2}{\lambda^{2} \mu^{2} \nu^{2} t}+\frac{t^{5}}{15120}+\cdots \\
& T(\lambda, \mu, \nu ; t)=\frac{t^{5}}{240}-\frac{17\left(\lambda^{2}+\mu^{2}+\nu^{2}\right) t^{7}}{40320}+\cdots
\end{aligned}
$$

for small $t$, together with the relation

$$
C(\lambda, \mu, \nu ; t)+T(\lambda, \mu, \nu ; t)=2 C(\lambda, \mu, \nu ; 2 t) .
$$

We define

$$
\begin{aligned}
f(\lambda, \mu, \nu ; t) & :=\left\{\frac{1}{C(\lambda, \mu, \nu ; t)}+\frac{1}{T(\lambda, \mu, \nu ; t)}\right\} \\
& =\frac{240}{t^{5}}+\frac{170\left(\lambda^{2}+\mu^{2}+\nu^{2}\right)}{7 t^{3}}+\cdots \\
F(\lambda, \mu, \nu ; L) & :=\frac{1}{f(\lambda, \mu, \nu ; L / 2)}=\frac{L^{5}}{7680}-\frac{17\left(\lambda^{2}+\mu^{2}+\nu^{2}\right) L^{7}}{5160960}+\cdots,
\end{aligned}
$$

noticing that for $\delta \in \mathbb{R}^{+}$we have the scaling formulae

$$
\begin{aligned}
C(\lambda, \mu, \nu ; t) & =\delta^{5} C(\delta \lambda, \delta \mu, \delta \nu ; t / \delta), & T(\lambda, \mu, \nu ; t) & =\delta^{5} T(\delta \lambda, \delta \mu, \delta \nu ; t / \delta) \\
f(\lambda, \mu, \nu ; t) & =\delta^{-5} f(\delta \lambda, \delta \mu, \delta \nu ; t / \delta), & F(\lambda, \mu, \nu ; t) & =\delta^{5} F(\delta \lambda, \delta \mu, \delta \nu ; t / \delta) .
\end{aligned}
$$

Finally we state, for each fixed triple $\lambda, \mu, \nu$ :

Hypothesis $\mathrm{A}(\lambda, \mu, \nu)$. The function $f(\lambda, \mu, \nu ; t)$ is convex for $t \in \mathbb{R}^{+}$.

This seems particularly awkward to prove and it is the sticking point in our method. A consequence of the hypothesis is that $f(\lambda, \mu, \nu ; t)$ is decreasing, as it converges to a finite limit as $t \rightarrow \infty$. Thus $F(\lambda, \mu, \nu ; t)$ increases with $t$.

REMARK 1. The scaling formulae (24) show that $\mathrm{A}(\lambda, \mu, \nu)$ and $\mathrm{A}(\delta \lambda, \delta \mu, \delta \nu)$ are equivalent for every $\delta>0$. So we can normalize, for example by assuming that $\lambda=1$.

The key result required for our application is

TheOREm 2. Suppose that $\nu>\mu>\lambda>0$ are such that Hypothesis $\mathrm{A}(\lambda, \mu, \nu)$ is valid. Then the conjectural inequality (13) holds for every function $y$ satisfying the conditions stated above together with (12). 
3. An easier extremal problem. In this section we tackle a supplementary problem, which we can solve completely, essentially by moving the maximum to one end of the interval.

THEOREM 3. Suppose that $y(x)$ is real-valued and three times differentiable on $[0, t]$, that $y^{\prime \prime \prime} \in L^{2}[0, t]$, and that $y(0)=M$ and $y^{\prime}(0)=y(t)=$ $y^{\prime}(t)=0$. Then if $\lambda, \mu, \nu$ are distinct positive numbers we have

$$
\begin{aligned}
& \int_{0}^{t}\left\{y^{\prime \prime \prime}(x)^{2}+\left(\lambda^{2}+\mu^{2}+\nu^{2}\right) y^{\prime \prime}(x)^{2}\right. \\
& \left.+\left(\lambda^{2} \mu^{2}+\mu^{2} \nu^{2}+\nu^{2} \lambda^{2}\right) y^{\prime}(x)^{2}+\lambda^{2} \mu^{2} \nu^{2} y(x)^{2}\right\} d x \geq \frac{1}{2} f(\lambda, \mu, \nu ; t) M^{2} .
\end{aligned}
$$

Notice that we do not require $M$ to be the maximum value of $|y|$ here, but it is intuitive that it actually is so in the extremal case, moreover that $y$ is then positive and decreasing, with $y^{\prime \prime}$ changing from negative to positive at some point of the interval. We do not assume any of these propositions.

Proof of Theorem 3. Denote the integral on the left of $(25)$ by $J(y)$. We expand $y^{\prime \prime \prime}(x)$ as a Fourier sine series on $[0, t]$ (with no claims about convergence). We may integrate this series term-by-term to obtain the Fourier cosine series of $y^{\prime \prime}(x)$, and we notice that there is no constant term, because $y^{\prime}(0)=y^{\prime}(t)$. Integrating term-by-term again we obtain the Fourier sine series of $y^{\prime}(x)$, and, after a final integration we have (say)

$$
y(x)=\frac{1}{2} a_{0}+a_{1} \cos \frac{\pi x}{t}+a_{2} \cos \frac{2 \pi x}{t}+a_{3} \cos \frac{3 \pi x}{t}+\cdots
$$

with equality in (26) because $y$ has bounded variation and is continuous. In particular, we have

$$
M=\frac{1}{2} a_{0}+a_{1}+a_{2}+a_{3}+\cdots, \quad 0=\frac{1}{2} a_{0}-a_{1}+a_{2}-a_{3}+\cdots,
$$

whence

$$
\frac{1}{2} M=\frac{1}{2} a_{0}+a_{2}+a_{4}+\cdots=a_{1}+a_{3}+a_{5}+\cdots .
$$

Put

$$
\begin{aligned}
b(n):= & \frac{\pi^{6} n^{6}}{t^{6}}+\left(\lambda^{2}+\mu^{2}+\nu^{2}\right) \frac{\pi^{4} n^{4}}{t^{4}} \\
& +\left(\lambda^{2} \mu^{2}+\mu^{2} \nu^{2}+\nu^{2} \lambda^{2}\right) \frac{\pi^{2} n^{2}}{t^{2}}+\lambda^{2} \mu^{2} \nu^{2} \\
= & \left(\frac{\pi^{2} n^{2}}{t^{2}}+\lambda^{2}\right)\left(\frac{\pi^{2} n^{2}}{t^{2}}+\mu^{2}\right)\left(\frac{\pi^{2} n^{2}}{t^{2}}+\nu^{2}\right),
\end{aligned}
$$


and observe that

$$
J(y)=\frac{t}{2}\left\{\frac{1}{2} b(0) a_{0}^{2}+\sum_{n=1}^{\infty} b(n) a_{n}^{2}\right\} .
$$

We apply Cauchy's inequality to each part of (28), to obtain

$$
\frac{1}{4} M^{2} \leq\left\{\frac{1}{2} b(0) a_{0}^{2}+b(2) a_{2}^{2}+b(4) a_{4}^{2}+\cdots\right\}\left\{\frac{1}{2 b(0)}+\frac{1}{b(2)}+\frac{1}{b(4)}+\cdots\right\}
$$

and

$$
\frac{1}{4} M^{2} \leq\left\{b(1) a_{1}^{2}+b(3) a_{3}^{2}+b(5) a_{5}^{2}+\cdots\right\}\left\{\frac{1}{b(1)}+\frac{1}{b(3)}+\frac{1}{b(5)}+\cdots\right\} .
$$

We deduce from (30)-(32) that

$$
\begin{aligned}
J(y) \geq & \frac{t}{8}\left\{\left(\frac{1}{2 b(0)}+\frac{1}{b(2)}+\frac{1}{b(4)}+\cdots\right)^{-1}\right. \\
& \left.+\left(\frac{1}{b(1)}+\frac{1}{b(3)}+\frac{1}{b(5)}+\cdots\right)^{-1}\right\} M^{2} .
\end{aligned}
$$

Recall that (except at the poles)

$$
\pi \operatorname{coth} \pi x=\frac{1}{x}+2 x \sum_{n=1}^{\infty} \frac{1}{n^{2}+x^{2}} .
$$

In order to employ (34) it is convenient to write $\lambda_{1}:=t \lambda / \pi$ etc., so that we have

$$
\begin{aligned}
\frac{1}{2 b(0)}+ & \frac{1}{b(2)}+\cdots \\
= & \frac{t^{6}}{\pi^{6}}\left\{\frac{1}{2 \lambda_{1}^{2} \mu_{1}^{2} \nu_{1}^{2}}+\sum_{\text {even } n \in \mathbb{N}} \frac{1}{\left(n^{2}+\lambda_{1}^{2}\right)\left(n^{2}+\mu_{1}^{2}\right)\left(n^{2}+\nu_{1}^{2}\right)}\right\} \\
= & \frac{t^{6}}{\pi^{6}}\left\{\frac{1}{\left(\mu_{1}^{2}-\lambda_{1}^{2}\right)\left(\nu_{1}^{2}-\lambda_{1}^{2}\right)}\left\{\frac{1}{2 \lambda_{1}^{2}}+\frac{1}{2^{2}+\lambda_{1}^{2}}+\frac{1}{4^{2}+\lambda_{1}^{2}}+\cdots\right\}\right. \\
& +\frac{1}{\left(\nu_{1}^{2}-\mu_{1}^{2}\right)\left(\lambda_{1}^{2}-\mu_{1}^{2}\right)}\left\{\frac{1}{2 \mu_{1}^{2}}+\frac{1}{2^{2}+\mu_{1}^{2}}+\frac{1}{4^{2}+\mu_{1}^{2}}+\cdots\right\} \\
& \left.+\frac{1}{\left(\lambda_{1}^{2}-\nu_{1}^{2}\right)\left(\mu_{1}^{2}-\nu_{1}^{2}\right)}\left\{\frac{1}{2 \nu_{1}^{2}}+\frac{1}{2^{2}+\nu_{1}^{2}}+\frac{1}{4^{2}+\nu_{1}^{2}}+\cdots\right\}\right\}
\end{aligned}
$$

by partial fractions. We write $\sum^{(3)}$ followed by the first of three terms to indicate a sum like (35) where the second and third terms are obtained by permuting the variables $\lambda_{1}, \mu_{1}, \nu_{1}$ cyclically. We apply (34), and recall (14), 
to find that the sum in (35) equals

$$
\begin{aligned}
\frac{t^{6}}{4 \pi^{5}} \sum(3) \frac{1}{\left(\mu_{1}^{2}-\lambda_{1}^{2}\right)\left(\nu_{1}^{2}-\lambda_{1}^{2}\right) \lambda_{1}} \operatorname{coth} \frac{\lambda_{1} \pi}{2} \\
=\frac{t}{4} \sum^{(3)} \frac{1}{\left(\mu^{2}-\lambda^{2}\right)\left(\nu^{2}-\lambda^{2}\right) \lambda} \operatorname{coth} \frac{\lambda t}{2} \\
=\frac{t}{4} C(\lambda, \mu, \nu ; t) .
\end{aligned}
$$

A similar argument involving (15) establishes that

$$
\begin{aligned}
\frac{1}{b(1)}+\frac{1}{b(3)} & +\frac{1}{b(5)}+\cdots \\
& =\frac{t}{4} \sum^{(3)} \frac{1}{\left(\mu^{2}-\lambda^{2}\right)\left(\nu^{2}-\lambda^{2}\right) \lambda} \tanh \frac{\lambda t}{2}=\frac{t}{4} T(\lambda, \mu, \nu ; t),
\end{aligned}
$$

and we insert (36) and (37) into (33) to obtain (25).

The inequality in (25) is sharp, moreover we may identify the extremal function as

$$
\begin{aligned}
y(x)= & \frac{M}{2} \sum^{(3)} \frac{1}{\left(\mu^{2}-\lambda^{2}\right)\left(\nu^{2}-\lambda^{2}\right) \lambda} \\
& \times\left\{\frac{\cosh \lambda(t / 2-x)}{C(\lambda, \mu, \nu ; t) \sinh (\lambda t / 2)}+\frac{\sinh \lambda(t / 2-x)}{T(\lambda, \mu, \nu ; t) \cosh (\lambda t / 2)}\right\} .
\end{aligned}
$$

To track this function down we observe that in the extreme case, each application of Cauchy's inequality in (31) and (32) must be sharp; that is to say, $a_{n}$ must be proportional to $1 / b(n)$ when $n$ is even or odd (or zero), but the constants of proportionality may be (and are) different in the various cases, and are determined by the boundary conditions $y(0)=M, y(t)=0$. It is easy to see in $(38)$ that $y^{\prime}(0)=y^{\prime}(t)=0$, as the hyperbolic functions cancel at the end-points when this expression is differentiated. This completes the proof.

REMARK 2. We expect the extremal function to be a linear combination of the six functions $e^{ \pm \lambda x}$ etc. because these are the independent solutions of the Euler-Lagrange equation associated with (25).

4. Proof of Theorem 2. We may assume that $a=0, b=L$. The boundary conditions are $y(0)=y(L)=y^{\prime}(0)=y^{\prime}(L)=0$ and we put

$$
\begin{aligned}
I(y):= & \int_{0}^{L}\left\{y^{\prime \prime \prime}(x)^{2}+\left(\lambda^{2}+\mu^{2}+\nu^{2}\right) y^{\prime \prime}(x)^{2}\right. \\
& \left.+\left(\lambda^{2} \mu^{2}+\mu^{2} \nu^{2}+\nu^{2} \lambda^{2}\right) y^{\prime}(x)^{2}+\lambda^{2} \mu^{2} \nu^{2} y(x)^{2}\right\} d x .
\end{aligned}
$$

Moreover we suppose that $|y(x)|$ attains its maximum value $M$ at the point $x=t$, indeed that $y(t)=M$, replacing $y$ by $-y$ if necessary. Then $y^{\prime}(t)=0$ 
and we see that we can split the integral in (39) into two ranges, $[0, t]$ and $[t, L]$, writing

$$
I(y)=: I_{1}(y)+I_{2}(y)
$$

On each range we have a problem of the type considered in Section 3. In the first range we apply Theorem 3 to the function $y(t-x)(0 \leq x \leq t)$ to deduce from (22) that

$$
I_{1}(y) \geq \frac{1}{2} f(\lambda, \mu, \nu ; t) M^{2},
$$

similarly we apply Theorem 3 to the function $y(x-t)(t \leq x \leq L)$ to obtain

$$
I_{2}(y) \geq \frac{1}{2} f(\lambda, \mu, \nu ; L-t) M^{2},
$$

whence

$$
I(y) \geq \frac{1}{2}\{f(\lambda, \mu, \nu ; t)+f(\lambda, \mu, \nu ; L-t)\} M^{2} .
$$

We do not know the value of $t$ and so we require the minimum of the righthand side as a function of $t$. On the assumption of Hypothesis $\mathrm{A}(\lambda, \mu, \nu)$ we see that this occurs in the middle of the range, that is, $I(y) \geq f(\lambda, \mu, \nu ; L / 2)$. We multiply this inequality by $F(\lambda, \mu, \nu ; L)$ as defined in (23) to deduce (13) as required. This completes the proof.

5. A special case. If the ratios $\lambda: \mu: \nu$ are rational then we can find $\kappa$ (and suppose it to be as large as possible) so that $\lambda, \mu$ and $\nu$ are integer multiples of $\kappa$, whence $\tanh (\lambda t / 2), \tanh (\mu t / 2), \tanh (\nu t / 2)$ are rational functions of $\tanh (\kappa t / 2)$. So therefore are $C(\lambda, \mu, \nu ; t)$ and $T(\lambda, \mu, \nu ; t) ;$ moreover if we put $\tanh (\kappa t / 2)=\tau$ then clearly $d t / d \tau$ is also a rational function of $\tau$. This means that in this case, Hypothesis $\mathrm{A}(\lambda, \mu, \nu)$ reduces to an elementary, if perhaps lengthy, calculus problem.

Consider the case $\mu=2 \lambda, \nu=3 \lambda$, in which $\kappa=\lambda$ and so

$$
\begin{aligned}
C(\lambda, 2 \lambda, 3 \lambda ; t) & =\frac{1}{\lambda^{5}}\left\{\frac{1}{24 \tau}-\frac{1}{30} \cdot \frac{1+\tau^{2}}{2 \tau}+\frac{1}{120} \cdot \frac{1+3 \tau^{2}}{3 \tau+\tau^{3}}\right\} \\
& =\frac{5-\tau^{2}}{60 \lambda^{5}\left(3 \tau+\tau^{3}\right)}, \\
T(\lambda, 2 \lambda, 3 \lambda ; t) & =\frac{1}{\lambda^{5}}\left\{\frac{\tau}{24}-\frac{1}{30} \cdot \frac{2 \tau}{1+\tau^{2}}+\frac{1}{120} \cdot \frac{3 \tau+\tau^{3}}{1+3 \tau^{2}}\right\} \\
& =\frac{2 \tau^{5}}{15 \lambda^{5}\left(1+\tau^{2}\right)\left(1+3 \tau^{2}\right)} .
\end{aligned}
$$

We have

$$
\begin{aligned}
C(\lambda, 2 \lambda, 3 \lambda ; t)^{-1}+T(\lambda, 2 \lambda, 3 \lambda ; t)^{-1} & \\
& =\frac{15 \lambda^{5}\left\{5+20 \tau^{2}+14 \tau^{4}+20 \tau^{6}+5 \tau^{8}\right\}}{2 \tau^{5}\left(5-\tau^{4}\right)},
\end{aligned}
$$


and we denote the right-hand side of $(45)$ by $\left(15 \lambda^{5} / 2\right) g(\tau)$. For this to be a convex function of $t$ it is necessary and sufficient that for $0<\tau<1$ we should have

$$
\left(1-\tau^{2}\right) g^{\prime \prime}(\tau)-2 \tau g^{\prime}(\tau)>0
$$

A calculation shows that

$$
-g^{\prime}(\tau)=\frac{5\left(1+\tau^{2}\right)\left(1-\tau^{2}\right)\left(25+60 \tau^{2}+30 \tau^{4}+12 \tau^{6}+\tau^{8}\right)}{\tau^{6}\left(5-\tau^{4}\right)^{2}}>0 .
$$

Now we differentiate (47) logarithmically to obtain

$$
\frac{g^{\prime \prime}(\tau)}{g^{\prime}(\tau)}=\frac{-4 \tau^{3}}{1-\tau^{4}}-\frac{6}{\tau}+\frac{8 \tau^{3}}{5-\tau^{4}}+\frac{120 \tau+120 \tau^{3}+72 \tau^{5}+8 \tau^{7}}{25+60 \tau^{2}+30 \tau^{4}+12 \tau^{6}+\tau^{8}} .
$$

The third and fourth terms increase on $[0,1]$ and so contribute at most 4.5 to the sum, whereas the first and second terms contribute less than -6 . Hence the right-hand side of (48) is negative and since $g^{\prime}(\tau)$ is also negative, we find that $g^{\prime \prime}(\tau)$ is positive, that is, both terms in (46) are positive. This is all we need.

6. Proof of Theorem 1. We suppose that $\lambda, \mu, \nu$ are such that Hypothesis $\mathrm{A}(\lambda, \mu, \nu)$ is valid and apply Theorem 2 with $y=Z^{2}, a=t_{n}$, $b=t_{n+1}=: a+l_{n}$. Since $F^{-1}=f$ by (23), we see that (13) yields

$$
\begin{array}{r}
M_{n}^{4} f\left(\lambda, \mu, \nu ; l_{n}\right) \leq \int_{t_{n}}^{t_{n+1}}\left\{\lambda^{2} \mu^{2} \nu^{2} Z(t)^{4}+\left(\lambda^{2} \mu^{2}+\mu^{2} \nu^{2}+\nu^{2} \lambda^{2}\right)\left\{\frac{d}{d t} Z(t)^{2}\right\}^{2}\right. \\
\left.+\left(\lambda^{2}+\mu^{2}+\nu^{2}\right)\left\{\frac{d^{2}}{d t^{2}} Z(t)^{2}\right\}^{2}+\left\{\frac{d^{3}}{d t^{3}} Z(t)^{2}\right\}^{2}\right\} d t .
\end{array}
$$

We add all these inequalities, to obtain

$$
\sum_{n=1}^{N} M_{n}^{4} f\left(\lambda, \mu, \nu ; l_{n}\right) \leq \int_{0}^{U}\left\{\lambda^{2} \mu^{2} \nu^{2} Z(t)^{4}+\cdots+\left\{\frac{d^{3}}{d t^{3}} Z(t)^{2}\right\}^{2}\right\} d t
$$

in which $U:=t_{N+1}$. Hardy and Littlewood [6] proved that $t_{N+1}-t_{N} \ll_{\varepsilon}$ $t_{N}^{1 / 4+\varepsilon}$ and so we have $T<U \leq T+T^{1 / 3}$ for large $T$. (In fact all we require in what follows is that $U \leq T+O(T / \log T)$.)

LEMMA 2. We have

$$
\begin{aligned}
& \int_{0}^{T}\left\{\frac{d^{k}}{d t^{k}} Z(t)^{2}\right\}^{2} d t \\
& =\frac{12}{(2 k+1)(2 k+2)(2 k+3)(2 k+4) \pi^{2}}\left\{1+O\left(\frac{1}{\log T}\right)\right\} T \log ^{2 k+4} T
\end{aligned}
$$

for each $k=0,1, \ldots$ as $T \rightarrow \infty$. 
We shall not prove this result here, but remark that we have derived the form of the main term from Conrey's formula [1], which is purely asymptotic, and relied on the method set out in [3] to provide an error term.

Put $\lambda=u \log T, \mu=v \log T, \nu=w \log T$, and recall that $\mathrm{A}(\lambda, \mu, \nu)$ and $\mathrm{A}(u, v, w)$ are equivalent. Then (50) and (51) give

$$
\begin{aligned}
\sum_{n=1}^{N} M_{n}^{4} f(\lambda, \mu, \nu & \left.l_{n}\right) \leq \frac{12}{\pi^{2}}\left\{\frac{u^{2} v^{2} w^{2}}{24}+\frac{u^{2} v^{2}+v^{2} w^{2}+w^{2} u^{2}}{360}\right. \\
+ & \left.\frac{u^{2}+v^{2}+w^{2}}{1680}+\frac{1}{5040}\right\}\left\{1+O\left(\frac{1}{\log T}\right)\right\} T \log ^{10} T
\end{aligned}
$$

By the scaling formulae (24), we have $f\left(\lambda, \mu, \nu ; l_{n}\right)=f\left(u, v, w ; l_{n} \log T\right) \log ^{5} T$. Since $f$ decreases, we have $f(u, v, w ; 2 \pi \theta) \leq f\left(u, v, w ; l_{n} \log T\right)$ whenever $l_{n} \leq 2 \pi \theta / \log T$ and so we may deduce from (52) that

$$
\sum_{n \leq N}\left\{M_{n}^{4}: l_{n} \leq \frac{2 \pi \theta}{\log T}\right\}
$$

$$
\begin{aligned}
\leq & \frac{1}{2 \pi^{2}} F(u, v, w ; 2 \pi \theta)\left\{u^{2} v^{2} w^{2}+\frac{u^{2} v^{2}+v^{2} w^{2}+w^{2} u^{2}}{15}+\frac{u^{2}+v^{2}+w^{2}}{70}+\frac{1}{210}\right\} \\
& \times\left\{1+O\left(\frac{1}{\log T}\right)\right\} T \log ^{5} T .
\end{aligned}
$$

We also have

$$
\begin{aligned}
\sum_{n \leq N} M_{n}^{4} \leq & \frac{1}{2 \pi^{2}} F(u, v, w ; \infty)\left\{u^{2} v^{2} w^{2}+\frac{u^{2} v^{2}+v^{2} w^{2}+w^{2} u^{2}}{15}\right. \\
& \left.+\frac{u^{2}+v^{2}+w^{2}}{70}+\frac{1}{210}\right\}\left\{1+O\left(\frac{1}{\log T}\right)\right\} T \log ^{5} T
\end{aligned}
$$

where, from (16), (22) and (23),

$$
F(u, v, w ; \infty):=\frac{u+v+w}{2 u v w(u+v)(v+w)(w+u)} .
$$

At this point our method is restricted by the fact that we have verified Hypothesis $\mathrm{A}(\lambda, \mu, \nu)$ in the case $1: 2: 3$ only. We put $v=2 u, w=3 u$ and obtain

$$
\sum_{n \leq N}\left\{M_{n}^{4}: l_{n} \leq \frac{2 \pi \theta}{\log T}\right\} \leq h(\theta, u)\left\{1+O\left(\frac{1}{\log T}\right)\right\} T \log ^{5} T,
$$

where

$$
h(\theta, u)=\frac{1}{2 \pi^{2}} F(u, 2 u, 3 u ; 2 \pi \theta)\left\{36 u^{6}+\frac{49}{15} u^{4}+\frac{1}{5} u^{2}+\frac{1}{210}\right\} .
$$


In the unrestricted case (55) implies

$$
h(\infty, u)=\frac{1}{240 \pi^{2}}\left\{36 u+\frac{49}{15 u}+\frac{1}{5 u^{3}}+\frac{1}{210 u^{5}}\right\} .
$$

The next step is to choose $u=u(\theta)$ to minimize $h(\theta, u)$ for each fixed $\theta \in \mathbb{R}^{+}$ and for $\theta=\infty$ : we find that $u(\infty)=.44061115 \ldots$, which yields $H_{2}(\theta)=$ $.010934581 \ldots$. just marginally better than the $.011113587 \ldots$ obtained in [5].

Let us turn to (56). From the definitions (14) and (15), we have

$$
\begin{aligned}
& C(u, 2 u, 3 u ; \pi \theta) \\
& \quad=\left\{\frac{1}{24} \operatorname{coth} \frac{\pi u \theta}{2}-\frac{1}{30} \operatorname{coth} \pi u \theta+\frac{1}{120} \operatorname{coth} \frac{3 \pi u \theta}{2}\right\} u^{-5} \sim \frac{1}{18 u^{6} \pi \theta}, \\
& T(u, 2 u, 3 u ; \pi \theta) \\
& \quad=\left\{\frac{1}{24} \tanh \frac{\pi u \theta}{2}-\frac{1}{30} \tanh \pi u \theta+\frac{1}{120} \tanh \frac{3 \pi u \theta}{2}\right\} u^{-5} \sim \frac{\pi^{5} \theta^{5}}{240},
\end{aligned}
$$

for fixed $\theta \in \mathbb{R}^{+}$and $u \rightarrow 0$. From (22) and (23),

$$
F(u, 2 u, 3 u ; 2 \pi \theta) \sim \frac{\pi^{5} \theta^{5}}{240}\left\{1-\frac{17}{24} \pi^{2} \theta^{2} u^{2}+\cdots\right\} \quad\left(\theta \in \mathbb{R}^{+}, u \rightarrow 0\right),
$$

whence from (57),

$$
h(\theta, 0)=\frac{\pi^{3} \theta^{5}}{100800} \quad\left(\theta \in \mathbb{R}^{+}\right) .
$$

We insert this into (56) to obtain the first part of Theorem 1.

Now we consider the minimization problem in (56). Put

$$
\tau:=\tanh \frac{\pi u \theta}{2}
$$

so that from (45), we have

$$
F(u, 2 u, 3 u ; 2 \pi \theta)=\frac{2 \tau^{5}\left(5-\tau^{4}\right)}{15 u^{5}\left(5+20 \tau^{2}+14 \tau^{4}+20 \tau^{6}+5 \tau^{8}\right)} \quad(0 \leq \tau \leq 1)
$$

and

$$
\begin{aligned}
& h(\theta, u) \\
& =\frac{\tau^{5}\left(5-\tau^{4}\right)}{15 \pi^{2}\left(5+20 \tau^{2}+14 \tau^{4}+20 \tau^{6}+5 \tau^{8}\right)}\left\{36 u+\frac{49}{15 u}+\frac{1}{5 u^{3}}+\frac{1}{210 u^{5}}\right\} .
\end{aligned}
$$

As $u$ increases, $\tau$ increases and so does the rational function of $\tau$ in (65) (see the proof of $\mathrm{A}(1,2,3)$ above). So we must choose $u$ in the range where the second factor in (65) decreases, say $0 \leq u \leq u_{2}$. Notice that $u_{2}=u(\infty)=$ $.44061115 \ldots$ 
We differentiate $h(\theta, u)$ logarithmically with respect to $u$ and find that

$$
\begin{aligned}
u & \frac{h^{\prime}(\theta, u)}{h(\theta, u)} \\
= & \left\{5+\frac{4 \tau^{4}}{5-\tau^{4}}-\frac{40 \tau^{2}+56 \tau^{4}+120 \tau^{6}+40 \tau^{8}}{5+20 \tau^{2}+14 \tau^{4}+20 \tau^{6}+5 \tau^{8}}\right\}\left(1-\tau^{2}\right) \frac{\operatorname{arctanh} \tau}{\tau} \\
& -\frac{5+126 u^{2}+686 u^{4}-7560 u^{6}}{1+42 u^{2}+686 u^{4}+7560 u^{6}} \\
= & : \psi(\tau)-\phi(u) \quad \text { (say), }
\end{aligned}
$$

and $h(\theta, u)$ is decreasing if $\phi(u)>\psi(\tau)$. It is easy to see by differentiation that $\phi(u)$ decreases from 5 to 0 on the range $\left[0, u_{2}\right]$, also we have $\psi(0)=5$, $\psi(1-0)=0$; we claim that $\psi(\tau)$ decreases on $[0,1]$, but this is a little awkward. First we observe that the right-hand factor $\left(1-\tau^{2}\right) \tau^{-1} \operatorname{arctanh} \tau$ decreases: if $\tau=\tanh \xi$ it equals $2 \xi / \sinh 2 \xi$, which clearly decreases. We write the left-hand factor in the form $5-4 m(x)$ where $x=\tau^{2}$ and

$$
m(x)=\frac{50 x+65 x^{2}+120 x^{3}+22 x^{4}-50 x^{5}-15 x^{6}}{\left(5-x^{2}\right)\left(5+20 x+14 x^{2}+20 x^{3}+5 x^{4}\right)},
$$

so that $m(0)=0, m(1)=3 / 4$. After a calculation we find that

$$
\begin{aligned}
\Delta x \frac{m^{\prime}(x)}{m(x)}= & 10(1-x)\left(125+450 x+1675 x^{2}+3495 x^{3}\right. \\
& \left.+3625 x^{4}+1943 x^{5}+777 x^{6}+197 x^{7}+6 x^{8}-5 x^{9}\right)
\end{aligned}
$$

where $\Delta$ denotes the denominator of $m^{\prime}(x) / m(x)$, that is, the product of the three factors in (67). We see from (68) that $m(x)$ increases on $[0,1]$ and so $5-4 m(x)$ decreases. Thus $\psi(\tau)$ decreases as required. We deduce that $u=\phi^{-1}\{\psi(\tau)\}$ is a one-to-one function mapping [0,1] onto [0, $\left.u_{2}\right]$.

We now simplify our calculations: rather than solve the equation $g^{\prime}(\theta, u)$ $=0$ for fixed $\theta$ we compute $\psi(\tau)$ for a range of values of $\tau$, as in Table 1 , and then solve the equation $\phi(u)=\psi(\tau)$ for $u$. (This is a cubic equation for $u^{2}$, suitable for a calculator: it is an easy matter to enhance its output by two or three significant figures.) Then we have, for this $\tau$ and $u$,

$$
\theta=\frac{1}{\pi u} \log \left(\frac{1+\tau}{1-\tau}\right)
$$

which yields the results listed in Table 1 . Notice that $\tau$ and $u$ tend to 0 together and $\theta$ converges to a limit $\theta_{2}$ which is computed by comparing the Maclaurin expansions of $\psi(\tau)$ and $\phi(u)$. We find that $\theta_{2}=6 \sqrt{14} / \pi \sqrt{17}$; for $\theta \leq \theta_{2}$ we cannot improve on (62). The graph of $H_{2}(\theta)$ must change from convex to concave beyond (if not at) $\theta_{2}$ and indeed flatten off pretty quickly: this is demonstrated by the table. 
A point of caution is that we have not demonstrated that for the values of $\theta$ obtained in this calculation we have actually found the optimal $u$ : certainly we have $\psi(\tau)-\phi(u)=0$ by construction, but it is conceivable that this is not the only local minimum of $h(u, \theta)$. However the method leads to an upper bound $H_{2}(\theta)$ as required and I preferred it to a computer search for a minimum.

\section{References}

[1] J. B. Conrey, The fourth moment of derivatives of the Riemann zeta-function, Quart. J. Math. Oxford Ser. (2) 39 (1988), 21-36.

[2] J. B. Conrey and A. Ghosh, A mean value theorem for the Riemann zeta-function at its relative extrema on the critical line, J. London Math. Soc. (2) 32 (1985), 193-202.

[3] R. R. Hall, The behaviour of the Riemann zeta-function on the critical line, Mathematika 46 (1999), 281-313.

[4] - , A Wirtinger type inequality and the spacing of the zeros of the Riemann zetafunction, J. Number Theory 93 (2002), 235-245.

[5] - On the extreme values of the Riemann zeta-function between its zeros on the critical line, J. Reine Angew. Math. 560 (2003), 29-41.

[6] G. H. Hardy and J. E. Littlewood, Contributions to the theory of the Riemann zetafunction and the theory of the distribution of primes, Acta Math. 41 (1917), 119-196.

[7] J. Steuding, Simple zeros and extreme values of the Riemann zeta-function on the critical line with respect to the zero-spacing, to appear.

Mathematics Department

York University

York YO10 5DD

United Kingdom 